\title{
Dominant-subordinate social interactions and subordinate behavioral responses in the primitively eusocial sweat bee Augochlora phoemonoe (Hymenoptera: Halictidae)
}

\author{
Milagros Dalmazzo $^{1,2}$, Arturo RoIG-Alsina ${ }^{1}$ \\ ${ }^{1}$ CONICET, División Entomología, Museo Argentino de Ciencias Naturales "Bernardino Rivadavia”, Av. Ángel \\ Gallardo 470, 1405, Buenos Aires, Argentina \\ ${ }^{2}$ CONICET, Departamento de Ciencias Naturales, Entomología, Facultad de Humanidades y Ciencias, Universidad \\ Nacional del Litoral, Paraje "El Pozo" s/n, 3000, Santa Fe, Argentina
}

Received 26 October 2017 - Revised 26 June 2018 - Accepted 20 September 2018

\begin{abstract}
Social interactions are studied in colonies of eusocial Augochlora phoemonoe, reared in artificial nests in the laboratory. Three kinds of behavioral interactions are distinguished between foundresses and daughter bees: antennation-tarsation (the most frequent), passing, and tandem-running following. Most interactions were started by the foundresses towards daughter bees. First-brood daughter bees displayed high frequencies of specific responses, indicating that these interactions function as behavioral mechanisms of colony integration in this eusocial augochlorine. Antennation-tarsation stimulated daughter females to collect pollen in a high percentage of cases and to get involved in construction activities in a lower percentage. After passing, daughter bees began nest construction activities in a high percentage of cases, and after tandem-running following, they were induced to guard the nest in a high percentage of cases. Locomotion had no specific relationship with any interaction. The behavioral responses were not related to the age of daughter females.
\end{abstract}

\section{Social interactions / Colony integration / Dominance behavior / Augochlora phoemonoe}

Eusocial bees, those with a high reproductive skew, division of labor, and alloparental care, have two modes of colony integration. Highly eusocial bees, such as the honeybee, rely on chemical signals to control the reproductive behavior of subordinates and integrate the activities of the colony (Hoover et al. 2003; Slessor et al. 2005; Nunes et al. 2010). In primitively eusocial bees, although some type of chemical communication could operate (Breed et al. 1978; Greenberg 1988; Rehan and Richards 2013), colony integration is achieved by

Electronic supplementary material The online version of this article (https://doi.org/10.1007/s13592-018-0609-8) contains supplementary material, which is available to authorized users.

Corresponding author: M. Dalmazzo,

milidalmazzo@yahoo.com

Manuscript editor: Yves Le Conte means of behavioral interactions between dominant and subordinate individuals. Halictid bees belong to this second class, where the outcome of social competition is largely governed by physical interactions (reviewed in Michener 1990; Pabalan et al. 2000; Smith et al. 2009; Kapheim et al. 2016).

Early in the study of sociality of Halictini bees, it was recognized that bees displayed particular behaviors in their interactions, the frequency of which was indicative of the social status of those bees in the colony. Some of the components of this behavioral repertoire were performed predominantly by the dominant individual and functioned in establishing reproductive dominance and colony integration (Brothers and Michener 1974; reviewed in Michener 1990). These interactions among individuals are considered as the appropriate units of analysis for understanding social phenomena (Arneson and Wcislo 2003; Jeanson et al. 2005). 
Social interactions are best known in Lasioglossum zephyrum, which has been model species regarding interactions in halictid bees. Its behavioral interactions have been studied in the context of kin recognition, nest defense, division of labor, and formation of dominance hierarchies (Bell 1974; Bell and Hawkins 1974; Brothers and Michener 1974; Breed 1977; Breed and Gamboa 1977; Kukuk et al. 1977; Breed et al. 1978; Kukuk 1980; Greenberg and Buckle 1981; Buckle 1982a, b, 1984; Greenberg 1988; Michener 1990). The emerging picture of this body of work is that interactions between dominant and subordinate individuals function as major mechanisms leading to social dominance, inhibition of ovary development, task allocation, and inhibition of mating. Ritualized aggression is thought to mediate the process of social competition. Information for other social halictids is fragmentary, and in many cases agonistic interactions, or the lack of them, have been observed in circle tube experiments using non-nestmate bees (e. g., Smith and Weller 1989; Pabalan et al. 2000; Arneson and Wcislo 2003; Packer 2006; Polidori and Borruso 2012). Interactions within observation nests have been studied in the facultatively eusocial Megalopta genalis in the context of trophallaxis and of the establishment of dominance relationships in early stage nests (Wcislo and Gonzalez 2006; Kapheim et al. 2016). Studies concerned with interactions during the life span of colonies, and with behavioral responses to different kinds of interactions, were lacking for halictid bees other than L. zephyrum .

Laboratory studies of Lasioglossum zephyrum have suggested that the dominant individual has a direct behavioral involvement in controlling worker tasks in the colony. Breed and Gamboa (1977) found that dominant bees performed a characteristic backing behavior when a pollen-laden forager entered the nest, leading the forager to a cell being provisioned, or after meeting the forager, backed for a short distance, and then turned and ran to the cell being provisioned stopping just below its entrance. In a similar study on the same species (Buckle 1984), the frequency of dominants directing worker behavior was considerably less, the pollen-laden workers being frequently able to locate cells without any assistance from the dominant. Buckle (1984) concluded that to assert the influence of the dominant bee on the behavior of workers, new experiments and/or long-term observations were necessary. Since then, this topic has remained without further analyses. We here address the question of whether the dominant bee is involved, and to which extent, in directing subordinate activities in colonies of Augochlora phoemonoe.

Augochlora (Augochlora) phoemonoe (Schrottky) is one of the five Augochlora present in temperate areas of southern South America (Dalmazzo and Roig-Alsina 2011). This species has wood-dwelling habits, a characteristic shared with all other species in the subgenus Augochlora (Stockhammer 1966; Michener 2007). Nests are rather shallow, with a short entrance tunnel. Cells are made with aggregated particles of chewed decaying wood, usually arranged in compact clusters of cells supported by pillars in a surrounding cavity. A. phoemonoe bees form primitively eusocial colonies with a cycle characteristic of temperate zone halictids. The nest is initiated by a solitary overwintered female. The first brood reared by the foundress consists solely of females, which remain in the nest as helpers to produce subsequent broods. Except for minor differences in size, there is no morphological differentiation between castes. There is a high reproductive skew, the founding female monopolizing oviposition, and the colony is characterized by a marked division of labor (Dalmazzo and Roig-Alsina 2015).

In this contribution, we analyze the social interactions between dominant and subordinate individuals, and the ensuing behaviors, in colonies of $A$. phoemonoe reared in artificial nests in the laboratory, from the birth of the first daughter bee to the decline of the colonies at the end of the season. We hypothesize that interactions have significant effects on the behavioral activities performed subsequently by subordinate bees. We predict that different kinds of interactions will affect the frequencies of the behavioral activities performed by subordinate bees as a response. 


\section{METHODS}

\subsection{Bee rearing}

The behavioral observations were conducted in artificial observation nests within a flight room set up to study the social biology of A. phoemonoe (Dalmazzo and Roig-Alsina 2015). The flight room, $2.0 \mathrm{~m} \times 2.5 \mathrm{~m}$ and $2.8-\mathrm{m}$ high, was furnished with 12 fluorescent $45 \mathrm{~W}$ tubes, lit $9 \mathrm{~h}$ a day (09:00-18:00 hours), to simulate the conditions of the woody environments where the species lives. The temperature was not controlled, but fluctuated according to conditions outdoors. Artificial nests were constructed following Michener and Brothers (1971), but modified to suit the needs of the studied species (Dalmazzo and Roig-Alsina 2015). They consisted of two glass panes $20 \times 30 \mathrm{~cm}, 1-\mathrm{cm}$ apart, and inserted into a wooden base. The two panes were filled with slices of decomposing wood of Salix sp., previously sterilized in a freezer, and covered with black plastic sheets to avoid light disturbing the nests. Most of the cell clusters, surrounding cavities, and tunnels of the nests constructed by the bees between the two closely set panes were accessible to the observer's sight, so that their behavior could be observed with minimal disturbance by periodically raising the black plastic sheets. Fresh flowers and diluted honey were provided daily for the bees flying in the room.

Eight eusocial nests were studied. These nests were initiated by solitary overwintered females which were collected in the surroundings of Buenos Aires in spring (September-October) 2008 and 2009. Each female was marked with a different color on the scutum and seeded into the Salix substrate between the glass panes. The nests became eusocial approximately 1 month later, with one to four daughter females. All bees of this first brood were females and stayed in the nests helping produce a second and a third brood. Firstbrood bees lived 75-125 days $(n=20, X=$ 109.15). Their ovary development was checked by dissection at the end of the season when the nests were dismantled, or as they died during the observation period, for a few of them. Colonies had a high reproductive skew, with $95 \%$ of firstbrood females with undeveloped ovaries. The study focuses on the interactions between the foundress and these first-brood females. A detailed description of the nesting biology of A. phoemonoe is in Dalmazzo and Roig-Alsina (2015). Daughter females were marked with a two-color code on the scutum: one color, the same color as the foundress, was used to indicate to which nest a female belonged, and the second one to discriminate between daughters of the same nest.

\subsection{Behavioral observations}

Nests were observed daily from the introduction of foundresses (September-October) until the end of the season (March). Each observation session was performed within the time frame of 08:00 to 20:00 hours. During each session, each nest was directly observed by the same observer continuously for $20 \mathrm{~min}$; the order in which the nests were observed was randomly assigned each day to avoid observation biases. Focal sampling was used to trace the behavior of individuals (using the scutum mark to discriminate between them). The number of times (frequency counts) that each individual started each interaction was recorded, as well as the ensuing behavior of the second individual. A total of 535 interactions and the corresponding following activities were recorded. Each interaction was scored only for the individual that started the interaction.

\subsection{Interactions}

Three types of interactions were observed: antennation-tarsation, passing, and tandemrunning (TR)-following. These interactions were recorded for all studied nests.

\subsubsection{Antennation-tarsation}

A bee approaches head-on another bee to a distance of $3 \mathrm{~mm}$ or less and then moves the antennae rapidly, touching antennae and head of the second individual (Online Resource 1). In most cases, the first bee also uses the fore tarsi to touch the head of the second individual. The second bee may respond moving also its antennae or staying still. After an antennation, the first bee 
may stay in place, back rapidly to the first position, or follow the receding bee. The bee that initiates the interaction may also approach the second bee from the back, touching with the antennae the third and fourth metasomal terga; the antennated bee stays still or moves away.

The approximation of the bees prior to the interaction may occur in two ways. One bee (usually the foundress) moves rapidly towards another bee, either from the front or the back, to a distance short enough to allow antennation. In other cases the foundress is still and the upcoming second bee suddenly stops at a distance of $8-10 \mathrm{~mm}$ of the foundress, which has the antennae extended forwards; then, the foundress rapidly moves forwards and antennation takes place. In some cases, a sequence of two or three rapid pulses of forward and backward movements occurs, with antennation-tarsation taking place each time the bees are close together.

\subsubsection{Passing}

Within the nest, a bee, either from the rear or the front, rapidly approaches another bee, which is still or slowly moving, and then moves past it. At passing, each bee swings $90^{\circ}$ to opposite walls of the tunnel, so the bees contact each other dorsum to dorsum. The foundress, when approaching head-on, may contact the daughter bee with the antennae before passing. The daughter bee may stay quiet for a few seconds after a passing.

\subsubsection{Tandem-running following}

An individual approaches another one frontally to a distance of $3 \mathrm{~mm}$ or less and then turns around and moves rapidly being closely followed by the second individual. The first individual begins the tandem-running (TR) and dictates the direction of the movement, either to the entrance or to the deep areas of the nest. The bees, separated by a distance no more than $3 \mathrm{~mm}$, travel $5-10 \mathrm{~cm}$ within the nest. TR-following ends when the first bee stops and turns around facing the second bee.

Interactions were registered in the nest cavities (tunnels and cavities surrounding the clusters). Prior to an interaction, in all cases, the individuals were inactive or in locomotion. No interactions were recorded while a bee was performing guarding, construction, or pollen collection. Thus, we were able to discriminate the activity responses as behaviors that occur after an interaction.

\subsection{Responses}

The activities following each interaction (responses) were classified into the following behavioral categories: locomotion (any displacement of the individual within the nest); guarding (at the nest entrance); construction (activities of tunneling, debris removal, and cell construction); and pollen collecting. All frequency counts of the observed interactions and responses were plotted in a "starting female/responding female" table (Online Resource 2).

\subsection{Data analysis}

Since data were not normally distributed, nonparametric statistics were employed. The significance of differences between foundress and daughter females regarding which kind of female started the interactions was tested with the MannWhitney $U$ statistical test. Chi-square tests were employed to analyze occurrence of each category of response for each type of interaction.

We used generalized linear mixed models (GLMM) with a binary distribution and a logit link function to evaluate the relationship between response and type of interaction. Each category of response (locomotion, guarding, construction, and pollen collecting) was considered as response variable $(1 / 0$, where $1=$ presence of the category of response following each interaction, and $0=a b-$ sence of this category). The interactions (antennation-tarsation, passing, and TR-following) and the age were considered as fixed factors. The division of the age of daughter females into four classes $(0=0$ to 20 days, $1=21$ to 40 days, $2=41$ to 60 days, and $3=$ more than 60 days, did not result in the GLMM model in any relationship between any of the four classes to the frequencies of the different categories of response performed by the daughter females. Because of this, we decided to use only two ages $(0=$ less than 40 days, and $1=$ more than 40 days) for GLMM 
analyses. The nest was the random factor. Through the inclusion of the nest as a random factor, we expected to obtain a more general picture, avoiding possible biases due to their peculiarities. The level of significance was $p<0.05$. For the statistical analyses, "Ime4" in the R program (http://www.R-project.org) was used.

\section{RESULTS}

In all the cases, the interactions involve two bees, and body contact may or may not occur. Most interactions (over $88 \%$ ) were started by the foundresses towards daughter bees (Figure 1). Differences between foundress $(n=8)$ and daughter $(n=20)$ bees regarding which caste started an interaction were statistically significant (MannWhitney test: $U=<0.001, p=0.001)$. A small percentage of interactions were started by daughter bees towards other daughter bees, two to nine interactions per nest. Higher frequencies occurred in nests with more daughter bees. The lowfrequency counts precluded a meaningful analysis of these data.

Antennation-tarsation was the most frequent interaction, followed by passing and TRfollowing (Table I). Foundresses interacted with all daughter bees in their nests, but daughter bees were never observed to initiate an interaction towards the foundress (Online Resource 2).

After each interaction started by the foundress, daughter bees performed different behaviors, the proportion of which varied according to the kind of interaction. The analysis of the behaviors showed consistent results across all nests (Table I and Online Resource 2).

The GLMM indicates that the type of interaction significantly affected the category of response performed by daughter females. On the contrary, there were no significant differences between the frequency of response of the two classes of age for any type of interaction (antennation-tarsation, GLMM: $t=-0.003, p=0.970$; passing, GLMM: $t=-0.514, p=0.607$; TR-following, GLMM: $t=-0.423, p=0.673$ ). To evaluate the possibility that young daughters may behave differently at an early age, we compared the responses to each kind of interaction of 0-10-day-old and more than 10 -day-old daughter females. A Mann Whitney $U$ test gave no significant differences between the two age classes for any kind of response $(U=$ 3211.50, $p=0.90$ for pollen collection; $U=$ 3049.50, $p=0.40$ for construction, and $U=$ $3153, p=0.60$ for guarding).

After antennation-tarsation, the behaviors of pollen collecting, construction, guarding, and locomotion were observed. The frequency of pollen collecting was significantly higher than the others (GLMM: $t=5.288, p<0.001$ ). Daughter females were more likely to collect pollen as a response after antennation-tarsation than to perform construction, guarding or locomotion (Figure 2).

After passing, daughter females were more likely to perform construction as a response than pollen collecting, guarding, or locomotion (Figure 2). The frequency of construction was significantly higher than the others (GLMM: $t=3.307$, $p<0.001)$.

After TR-following, daughter females were more likely to perform guarding as a response than pollen collecting or locomotion (Figure 2). The frequency of guarding was significantly higher than the others (GLMM: $t=2.893$, $p<0.001)$.

No interaction had a significant relationship to locomotion. Daughter females have a low probability of performing locomotion after any interaction (antennation-tarsation, GLMM: $t=0.642$, $p=0.521$; passing, GLMM: $t=0.422, p=$ 0.684; TR-following, GLMM: $t=0.414, p=$ 0.674).

The nest as random factor had no significant effect on the behaviors analyzed. The responses of the daughter females were similar regardless of the nest of origin (pollen collection, GLMM: $t=$ $0.248, p=0.162$; construction, GLMM: $t=$ $0.179, p=0.527$; guarding, GLMM: $t=0.106$, $p=0.435$; locomotion, GLMM: $t=0.095, p=$ $0.637)$.

\section{DISCUSSION}

Our data provide evidence of the behavioral involvement of the foundress in promoting and directing the tasks performed in colonies of a primitively eusocial Augochlorini. We found that different behaviors displayed by the foundress elicited differential responses by the daughter 

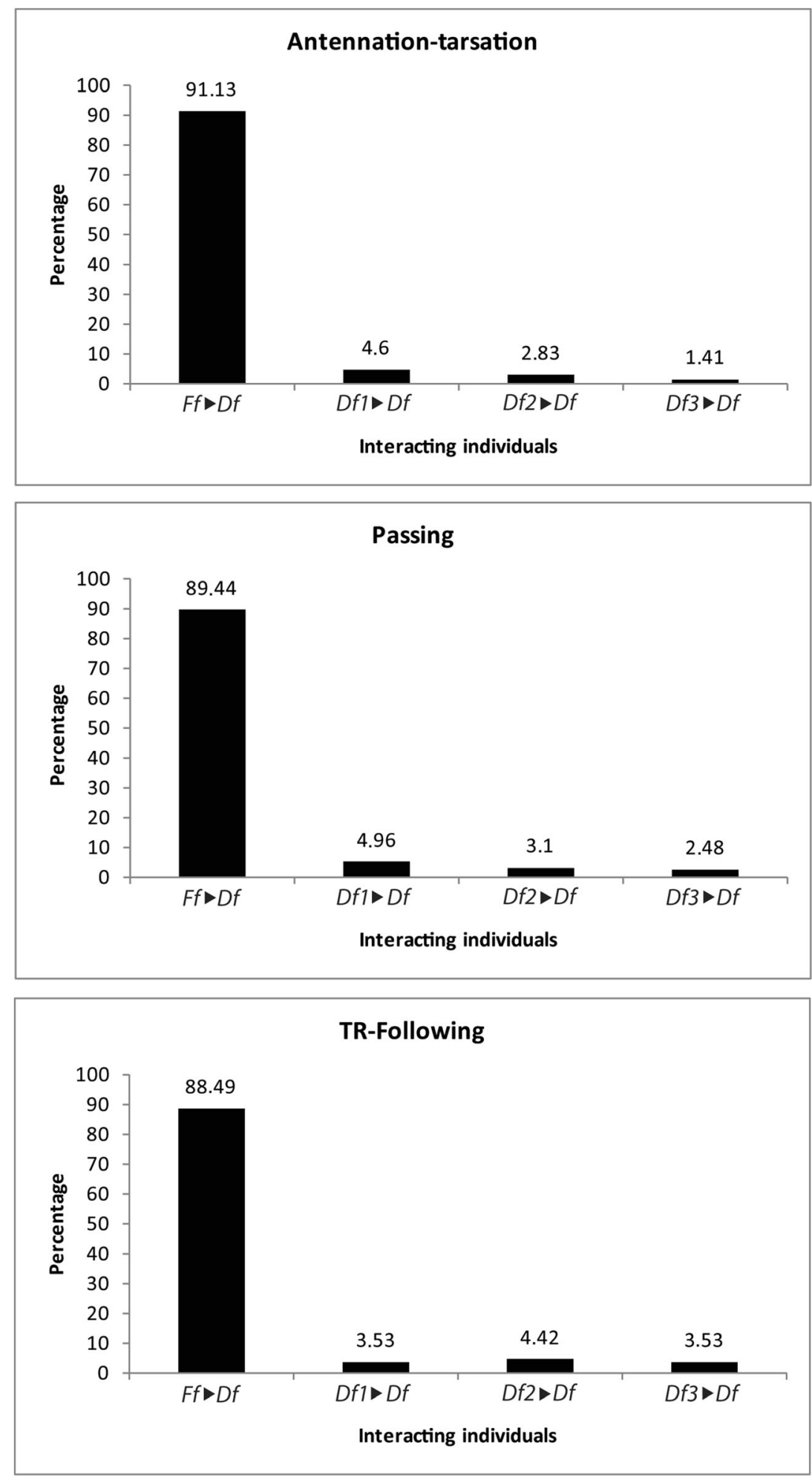
Table I.. Interactions and following responses in Augochlora phoemonoe. Responses are: Pollen collecting (Pc), Construction (Cn), Guarding (Gu), and Locomotion (Lo). Total count values for the eight nests are given. Categories of responses and type of interaction are not independent variables (chi-square $\chi^{2}$ test, $p \leq 0.001$ )

\begin{tabular}{lllllll}
\hline Interactions & & \multicolumn{2}{l}{ Responses } & & & \\
\cline { 3 - 7 } & & Pc & Cn & Gu & Lo & Total \\
\hline Antennation-tarsation & Count & $\mathbf{1 9 1}$ & 54 & 14 & 14 & 273 \\
Passing & Count & 7 & $\mathbf{1 3 5}$ & 9 & 4 & 155 \\
TR-following & Count & 1 & 0 & $\mathbf{9 4}$ & 12 & 107 \\
& $\chi^{2}$ & 268.28 & 271.78 & 307.65 & 7.82 & \\
& $d f$ & 2 & 2 & 2 & 2 & \\
& $p$ & 0.0001 & 0.0001 & 0.0001 & 0.02 & \\
\hline
\end{tabular}

Bold values indicate statistical significant differences between responses. Statistical values for chi-square, degree of freedom and $p$ are in Italics

bees. In the small colonies of $A$. phoemonoe, formed by the mother and her one to four daughters, the frequencies of initiation of interactions for the three behavioral patterns were maximal for the foundress. Daughter bees initiated interactions among themselves at very low frequencies and they were never observed to initiate an interaction towards the foundress.

The most frequent interaction was antennationtarsation. It can be interpreted as a ritualized agonistic behavior performed by the foundress. Its aggressiveness is particularly evident when bouts of rapid forward and backward movements of the foundress take place, each accompanied by antennation-tarsation (Online Resource 1). Our study suggests that the subordinate bee is coerced into abandoning an idling state and performing certain activities. Other, more overt aggressive interactions were not observed. Behaviors such as C-postures, with sting presentation, or threats of biting by opening the mandibles, were absent. This is reasonable, since interactions were observed among individuals of the same nest, where dominance relationships have already been established. The interaction antennation-tarsation may also involve chemical cues. Several studies

Figure 1. Interactions initiated by foundress females (Ff) towards daughter females (Df), and interactions initiated by Df towards other Df. Percentages based on total observations for all nests of $A$. phoemonoe reared in laboratory. Mann-Whitney $U$ test $(p=0.001)$ indicates statistically significant differences between Ff and Df. have shown that cuticular hidrocarbons are related to division of labor in social insects. In stingless bees, it has been shown that chemical profiles differ between groups of workers performing different tasks (Ferreira-Caliman et al. 2010). In ants of the genus Diacamma (Shimoji et al. 2017), the queen transfers to the workers a queen presence signal by direct contact, which regulates the physiological state of the workers. Although these aspects have not been studied in Augochlorini bees, a role of chemical cues in the maintenance of the dominance hierarchy cannot be excluded. However, regarding the regulation of the colony activities in A. phoemonoe, the fact that in the observation nests different interactions between the same individuals result in the performance of different tasks points to a behavioral mechanism of regulation. In the studied colonies antennationtarsation had a strong effect upon subordinates, inducing them to forage in a high percentage of cases, and to get involved in construction activities in a lower percentage. Initiation of passing behavior in A. phoemonoe is a prerogative of the foundress and may serve, as the former behavior, to impose its social status and direct activities in the colony. Subordinate bees began nest construction activities in a high percentage of cases as a response to passing. The third type of interaction, TR-following, also shows the preeminent role of the dominant individual. It was the least frequent interaction. Daughter bees were induced to guard the nest in a high percentage of cases. 

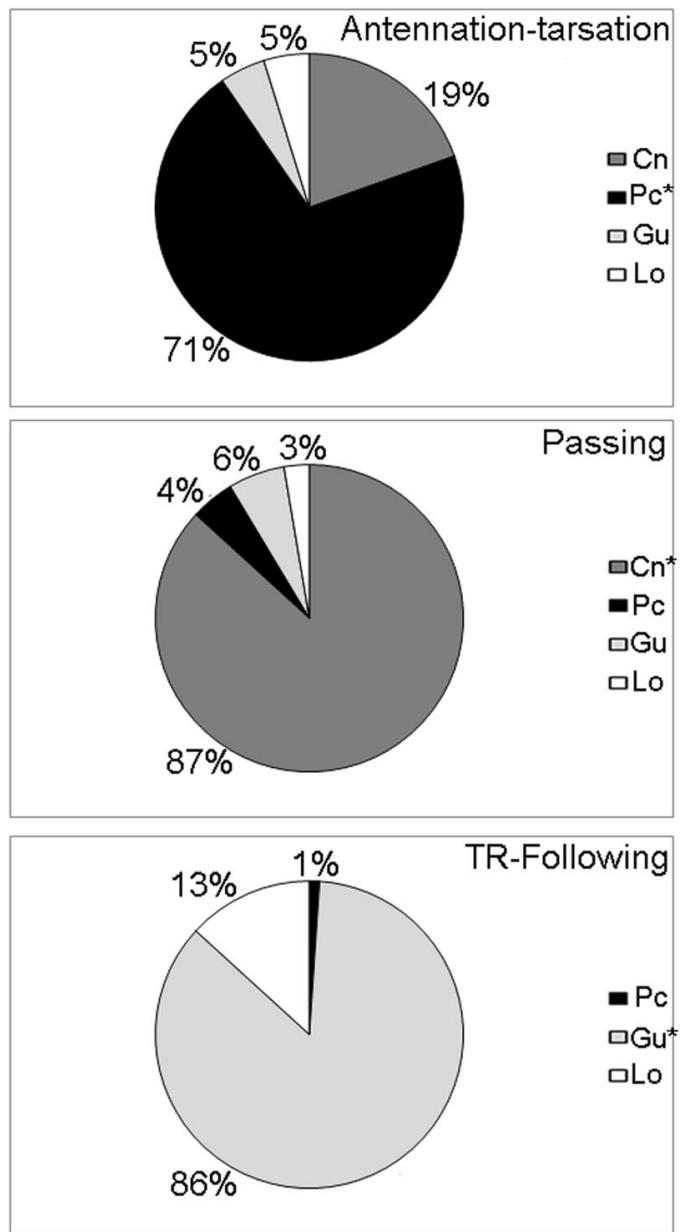

Figure 2. Responses performed by daughter females after the interactions antennation-tarsation, passing and TR-following. Percentages based on total observations for all nests of $A$. phoemonoe reared in laboratory. $\mathrm{Cn}=$ construction activities, $\mathrm{Pc}=$ pollen collecting, $\mathrm{Gu}=$ guarding, and Lo = locomotion. Asterisk indicates statistically significant differences (chi-square $\chi^{2}$ test, $p<$ $0.001)$.

We found no evidence of a temporal division of labor. Daughter bees performed all the tasks in the nest regardless of their age. In the GLMM model, age had no significant relationship to pollen collecting, construction, or guarding. In another Augochlorini, Megalopta genalis, task performance differs between recently born individuals and established workers (Kapheim et al. 2016). In M. genalis, the first
10 days after worker emergence are critical for the development of worker behavior. In the present study, we made no emphasis in the observation of callow individuals, and we thus would not have been able to detect an early division of tasks.

In the primitively eusocial L. zephyrum, it was hypothesized that the high level of activity of the queen and her specific behaviors play a direct role in integrating the colony (Michener and Brothers 1974). The high rate of nudging and backing of the queen (which is accompanied by following of other bees as a response) promotes increased activity of the colony members by drawing quiescent individuals down from the vicinity of the burrow entrance to the region where most of the work such as burrowing, cell construction, and cell provisioning occurs. The response of workers to the queen backing and nudging was found to be related to ovarian size of workers, those with somewhat enlarged ovaries tending to remain in the nest and guard (although they do sometimes forage) and those with more slender ovaries tending to work on cells and to forage to provision the cells (Brothers and Michener 1974). Consistent with what is known for L. zephyrum, we found a preeminent role of the dominant individual in promoting colony activity, but in A. phoemonoe, we found nearly no variance in ovarian development and no task specialization among workers.

Previous L. zephyrum studies (Breed and Gamboa 1977; Buckle 1984) found that pollen-laden bees entering the nest were guided by the queen, so the foragers were more likely to find the cells that were being provisioned. Such a direct behavioral involvement of the dominant individual in directing nest activities is in agreement with our findings in A. phoemonoe, where we found significant effects of the kind of interaction initiated by the dominant individual on the resulting tasks performed by the subordinate bees. However, experiments manipulating the bee encounters would be desirable to confirm if specific behavioral changes are causally related in the interactions between foundress and daughter bees. 


\section{ACKNOWLEDGMENTS}

The authors thank Rocío Gonzalez-Vaquero for her invaluable help in bee rearing, Leticia Zumoffen and Marcelo Signorini for helping in data analysis, and Lina Horovitz for providing comments on language style. The authors also thank two anonymous reviewers for their comments on the manuscript.

\section{AUTHOR CONTRIBUTION}

Both authors have contributed equally to the work.

\section{FUNDING}

This study was supported by grants ANPCyT, Argentina, 2007-1238, and CONICET, Argentina, PIP 2011-0288.

\section{COMPLIANCE WITH ETHICAL STANDARDS}

Conflict of interest The authors declare that they have no competing interests.

Interactions sociales dominantes-subordonnées et réponses comportementales subordonnées chez l'abeille primitive eusociale Augochlora phoemonoe (Hymenoptera: Halictidae).

Interactions sociales / intégration de colonies / comportement de dominance / Augochlora phoemonoe

Dominant-subordinate soziale Interaktionen und subordinate Verhaltensantwort bei der primitiv-eusozialen Furchenbiene Augochlora phoemonoe (Hymenoptera: Halictidae).

Soziale Interaktionen / Kolonieintegration / Dominanzverhalten / Augochlora phoemonoe

\section{REFERENCES}

Arneson, L., Wcislo, W. T. (2003) Dominant-subordinate relationship in a facultatively social, nocturnal bee, Megalopta genalis (Hymenoptera: Halictidae). J. Kans. Entomol. Soc. 76, 183-193
Bell, W. J. (1974) Recognition of resident and non-resident individuals in intraspecific nest defense of a primitively eusocial halictine bee. J. Comp. Physiol. 93, 195-202

Bell, W. J., Hawkins, W. A. (1974) Patterns of intraspecific agonistic interactions involved in nest defense of a primitively eusocial Halictine bee. J. Comp. Physiol. 93, 183-193

Breed, M. D. (1977) Interactions among individuals and queen replacement in a eusocial halictine bee. Proc. $8^{\text {th }}$ Internat. Cong., Internat, Union Stud. Soc. Insect (Wageningen)

Breed, M. D., Gamboa, G. J. (1977) Behavioral control of workers by queens in primitively eusocial bees. Science 195, 694-696

Breed, M. D., Silverman, J. M., Bell, W. J. (1978) Agonistic behavior, social interactions and behavioral specialization in a primitively eusocial bee. Ins. Soc. 25, 351-364

Brothers, D. J., Michener, C. D. (1974) Interactions in colonies of primitively social bees. III Ethometry of division of labor in Lasioglossum zephyrum (Hymenoptera: Halictidae). J. Comp. Physiol. 90, 129-168

Buckle, G. R. (1982a) Differentiation of queens and nesmate interactions in newly established colonies of Lasioglossum zephyrum (Hymenoptera: Halictidae). Sociobiology 7, 8-18

Buckle, G. R. (1982b) Queen-worker behavior and nesmates interactions in young colonies of Lasioglossum zephyrum. Ins. Soc. 29, 125-137

Buckle, G. R. (1984) A second look at queen-forager interactions in the primitively eusocial halictid, Lasioglossum zephyrum. J. Kans. Entomol. Soc. 57, 1-6

Dalmazzo, M., Roig-Alsina, A. (2011) Revision of the species of the New World genus Augochlora (Hymenoptera, Halictidae) occurring in the southern temperate areas of its range. Zootaxa $\mathbf{2 7 5 0 ,}$ $15-32$

Dalmazzo, M., Roig-Alsina, A. (2015) Social biology of Augochlora (Augochlora) phoemonoe (Hymenoptera: Halictidae) reared in laboratory nests. Ins. Soc. 62, 315-323

Ferreira-Caliman, M. J., Nascimento, F. S., Turatti, I. C., Mateus, S., Lopes, N. P., Zucchi, R. (2010) The cuticular hydrocarbons profiles in the stingless bees Melipona marginata reflect task-related differences. J. Insect. Physiol. 56, 800-804

Greenberg, L. (1988) Kin recognition in the sweat bee Lasioglossum zephyrum . Behav. Genet. 18, 425-438

Greenberg, L., Buckle, G. R. (1981) Inhibition of worker mating by queens in a sweat bee, Lasioglossum zephyrum. Ins. Soc. 28, 347-352

Hoover, S. E., Keeling, C. I., Winston, M. L., Slessor, K. N. (2003) The effect of queen pheromones on worker honey bee ovary development. Naturwissenschaften 90, 477-80

Jeanson, R., Kukuk, P. F., Fewell, J. H. (2005) Emergence of division of labour in halictine bees: contributions of 
social interactions and behavioural variance. Anim. Behav. 70, 1183-1193

Kapheim, K. M., Chan, T. Y., Smith, A. R., Wcislo, W. T., Nonacs, P. (2016) Ontogeny of division of labor in a facultatively eusocial sweat bee Megalopta genalis . Ins. Soc. 63, 185-191

Kukuk, P. F. (1980) Diurnal activity of a primitively eusocial bee, Lasioglossum zephirum, within the nest. J. Kans. Entomol. Soc. 53, 149-156

Kukuk, P. F., Breed, M. D., Bobti, A., Bell, W. (1977) The contribution of kinship and conditioning to nest recognition and colony member recognition in a primitively eusocial bee, Lasioglossum zephyrum. Behav. Ecol. Sociobiol. 2, 319-327

Michener, C. D. (1990) Reproduction and castes in social halictine bees, in: Engels W (Ed.) Social insects: an evolutionary approach to castes and reproduction. Springer, Berlin, pp. 77-121

Michener CD (2007) The bees of the world. $2^{\text {nd }}$ Ed. Johns Hopkins University Press, Baltimore Londres.

Michener, C. D., Brothers, D. J. (1971) A simplified observation nest for burrowing bees. J. Kans. Entomol. Soc. 44, 236-239

Michener, C. D., Brothers, D. J. (1974) Were worker of eusocial Hymenoptera initially altruistic or oppressed? Proc. Natl. Acad. Sci. U. S. A. 71, 671-674

Nunes, T. M., Morgan, E. D., Drijfhout, F. P., Zucchi, R. (2010) Caste-specific cuticular lipids in the stingless bee Friesella schrottkyi. Apidologie 41, 579-588

Pabalan, N., Davey, K. G., Packer, L. (2000) Escalation of aggressive interactions during staged encounters in Halictus ligatus say (Hymenoptera: Halictidae), with a comparison of circle tube behaviors with other halictine species. J. Insect. Behav. 13, 627-650
Packer, L. (2006) Use of artificial arenas to predict the social organization of halictine bees: data for fourteen species from Chile. Ins. Soc. 53, 307-315

Polidori, C., Borruso, L. (2012) Socially peaceful: foragers of the eusocial bee Lasioglossum malachurum are not aggressive against non-nestmates in circle-tube arenas. Acta. Ethol. 15, 15-23

Rehan, S. M., Richards, M. H. (2013) Reproductive aggression and nestmate recognition in a subsocial bee. Anim. Behav. 85, 733-741

Shimoji, H., Aonuma, H., Miura, T., Tsuji, K., Sasaki, K., Okada, Y. (2017) Queen contact and among-worker interaction dually suppress worker brain dopamine as a potential regulation of reproduction in ant. Behav. Ecol. Sociobiol. 71, 35

Slessor, K. N., Winston, M. L., Le Conte, Y. (2005) Pheromone communication in the honeybee (Apis mellifera L.). J. Chem. Ecol. 31, 2731-2745

Smith, B. H., Weller, C. (1989) Social competition among gynes in halictine bees: the influence of bee size and pheromones on behavior. J. Insect. Behav. 2, 397-411

Smith, A. R., Kaphein, K. M., O’Donnell, S., Wcislo, W. T. (2009) Social competition but not subfertility leads to a division of labour in the facultatively social sweat bee Megalopta genalis (Hymenoptera: Halictidae). Anim. Behav. 78, 1043-1050

Stockhammer, K. A. (1966) Nesting habit and life cycle of a sweet bee, Augochlora pura. J. Kans. Entomol. Soc. 39, 157-192.

Wcislo, W. T., Gonzalez, V. H. (2006) Social and ecological contexts of trophallaxis in facultatively social sweat bees, Megalopta genalis and M. ecuadoria (Hymenoptera: Halictidae). Ins. Soc. 53, 220-225 